\title{
On improving urban environment representations
}

\author{
Xavier Pueyo $^{1}$ *, Carles Bosch ${ }^{2}$ and Gustavo A. Patow ${ }^{1}$ \\ 1 Visualització, Realitat Virtual i Interacció Gràfica (ViRVIG), Universitat de Girona, Girona, Spain \\ 2 Barcelona Media, Barcelona, Spain
}

Edited by:

Daniel Thalmann, Nanyang

Technological University, Singapore

\section{Reviewed by:}

Jian Chen, University of Maryland

Baltimore County, USA

Mario Arturo Gutierrez, OZWE Sàrl,

Switzerland

Luciana Nedel, Universidade Federal

do Rio Grande do Sul, Brazil

*Correspondence:

Xavier Pueyo, Centre de Recerca

ViRVIG-UdG, Universitat de Girona,

Edifici P4, Campus de Montilivi,

Girona E-17071, Spain

e-mail:xavier.pueyo@udg.edu
Computer graphics has evolved into a mature and powerful field that offers many opportunities to enhance different disciplines, adapting to the specific needs of each. One of these important fields is the design and analysis of Urban Environments. In this article, we try to offer a perspective of one of the sectors identified in Urban Environment studies: Urbanization. More precisely, we focus on geometric and appearance modeling, rendering, and simulation tools to help stakeholders in key decision stages of the process.

Keywords: computer graphics, urban models, rendering, appearance, simulation, aging, urban physics

\section{INTRODUCTION}

An important key topic omnipresent in the $\mathrm{R}+\mathrm{D}$ agendas of national and international bodies is Urban Environment. Urban Environment encompasses many sectors. While it seems that here it is not an absolute agreement in how institutions identify the main problems, the following sectors are usually taken into account: Urbanization, Energy, Health, Mobility, Water, Sanitation, and Solid Waste. In turn, Urbanization includes many fields: technology, sociology, cultural heritage, among others.

Focusing in the technological context, computer graphics have witnessed an important evolution since the early digital cartography and CAD systems of the late 70s and the 80s. This evolution, together with the important increase in computing power (CPU and GPU) in the same time-frame and the availability of huge amounts of data, have enabled new computation and simulation possibilities, opening the door for a variety of research topics. Some of these topics arise as outstanding opportunities to improve the tools used in application areas that directly impacts virtual urban environments design, which in turn affects life quality in urban areas.

In this paper, we will focus on geometric and appearance modeling, rendering, and simulation tools for urban environments design and analysis trying to contribute to highlight some of their recent advances and challenges.

\section{URBAN MODELING}

A broad range of areas, such as games, movies or urban design require virtual $3 \mathrm{D}$ city models with detailed geometry. In particular, games have presented us with awesome urban landscapes, but in general these are created manually with hundreds of artists. Procedural modeling has proven to be effective for this task, offering a potential alternative to the labor-intensive modeling of traditional 3D techniques (Watson et al., 2008; Vanegas et al., 2010). In recent years, after the seminal works by Parish and Müller (2001) and
Müller et al. (2006), we have witnessed a blossom in inverse procedural techniques, in particular applied to the recovery, capture and modeling of plants, trees, and urban models (Smelik et al., 2014). Although the many improvements, there are still many open issues for future research, as described by Musialski et al. (2013) and Patow and Besuievsky (2013). Some of these challenges and open problems for urban modeling include better tools for capturing and handling data, improved editing tools, dealing with irregular architecture, and specially handling real-world architecture, like the works from Gehry or Gaudí. Advancing on these challenges will have a direct impact on fields like architecture, where architects have recently started to use parametric and procedural tools to conceive sophisticated buildings (e.g., Advances in Architectural Geometry, 2014). See Figure 1. Another example is GIS, where efforts are focused on extending current tools to incorporate 3D urban modeling, like in the case of Esri's acquisition of City Engine (http://www.esri.com/software/cityengine). A final area where we expect these techniques will have a profound impact is the new discipline of smart cities, where a careful combination of remote sensing technology, planning, networks and modeling tools could completely reshape the way planners conceive their tools (Aliaga, 2012).

Another area that has received little attention (Weber et al., 2009) is time-dependent geometric modeling, as buildings and cities are not static objects in time, but are in a continuous evolution that exists simultaneously at different time scales. These time scales range from years for aging effects (see Appearance Modeling), up to decades and even centuries for large-scale urban changes, like in the case of a cathedral or a castle. They undergo not only changes concerning the entire building life-cicle (construction, refurbishment, merging, demolition, etc.) but also local changes that only affect some elements, like the façade or its columns. We cannot doubt of the large impact such tools will have in the humanities, affecting mainly art, history, and archeology. 

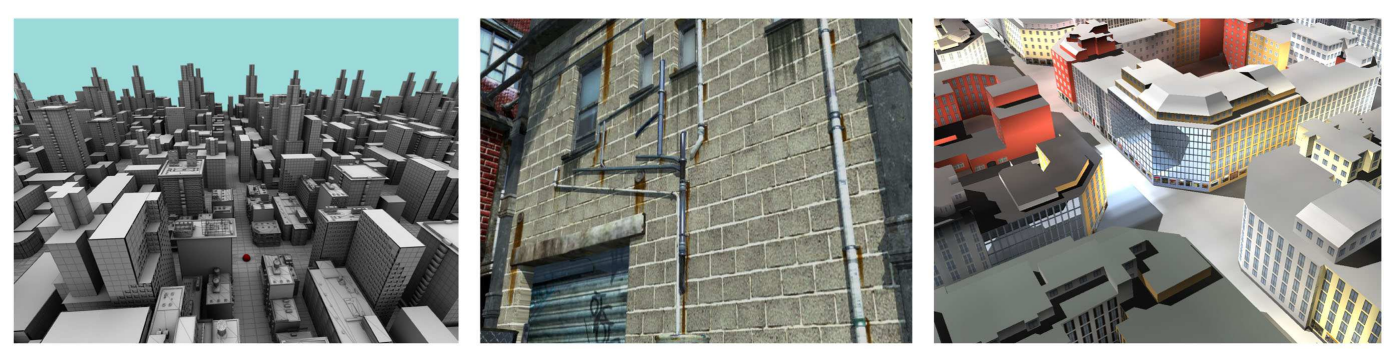

FIGURE 1 |Three examples of applications of computer graphics to urban environments. Left: Level-of-Detail, middle: weathered appearance, right: global illumination simulations.

Closely related, urbanism is another area with a growing interest in modeling techniques, as they allow both to reflect the current urban situation and to predict future changes, including the study of alternative courses of action, as shown by Vanegas et al. (2012).

\section{APPEARANCE MODELING}

Modeling urban environments not only means having precise geometric models but also appropriate material descriptions. In particular, representing the way materials interact with light, commonly referred as appearance, is key for generating realistic and convincing virtual city models.

One of the main difficulties in modeling material appearance is dealing with its high dimensionality, since it often involves changes over the spectral, directional, spatial, or even time domains. Urban models have the additional complexity of requiring hundreds or thousands of different materials along with their variations or textures. While recent efforts provide means to handle highly detailed models, they mostly consist of color information or simple reflection models (Kuang et al., 2013). Hence, there is a need to provide richer appearances to such environments.

Real material reflectance exhibits complex behaviors that are difficult to capture with existing analytical representations. The use of measured data has thus gained widespread in areas such as the automotive industry, but its adoption for large-scale scenarios still requires appropriate approximations. One area that has raised interest in the community is representing reflectance by means of statistical models, which has connections with perceptual studies on material appearance. Nishino and colleagues have demonstrated that they are good candidates for approximating data and for image-based estimation (Lombardi and Nishino, 2012). Further advances in this area are foreseen, since those descriptions need to be simplified in order to ease their manipulation and storage, as well as including more complex reflectance behaviors.

Incorporating temporal effects is also key for realistic environments. Most materials are affected by weathering and aging effects, which modify their appearance over time. Several tools are starting to appear to easily incorporate such effects for artists (Substance Painter, enRich), but completely lacking of physical accuracy. Simulation based on first principles is still computationally demanding, while capture-and-transfer methods are usually tied to the available exemplars. The use of historical data (e.g., in the form of photographs) has not been exploited yet, which could provide interesting insights on their appearance evolution.
On the other hand, hybrid solutions combining simulation with data-driven methods have only scratched its surface (Bosch et al., 2011). See Figure 1, middle. City models will require faster simulations, while the interplay between the environmental conditions and the presence of such effects stills needs of specific tools.

All data-driven methods require good acquisition approaches, and uncontrolled environments such as urban scenarios are in general problematic. Data is increasingly becoming available especially in the form of photographs, which can be exploited to recover appearance under several lighting and viewing conditions (Laffont et al., 2012). Image-based reconstruction and depth estimation is also gaining significance with the presence of new devices that would facilitate this task (Venkataraman et al., 2013; SCENE, 2014). Moreover, single-image acquisition is showing advances on recovering light and reflectance at the same time (Lombardi and Nishino, 2012). All these advances will open interesting possibilities for easily capturing data, especially from hand-held devices.

Appearance modeling has important implications in diverse areas. In architecture and industrial design, it provides appealing images for costumers but also facilitates understanding for architects or interior designers. Other areas that would benefit from advances on this area include virtual prototyping, simulation and training, entertainment, and cultural heritage (Dorsey and Rushmeier, 2008).

\section{RENDERING}

Computer generated images are useful for human beings and machines. In the first case, the images are intended to help the comprehension of the model (Vanegas et al., 2010). Concerning machines, the synthetic images could be used to train and calibrate vision systems. To the best of our knowledge, this has not yet been studied in depth. We think it could be an important direction for further research in order to reduce the computational cost by means of replacing real testing environments by virtual ones (digital maquettes). An example of this Šlosár et al. (2014) is the design and test of high quality cameras for traffic control or security as well as for design and test of vision systems for autonomous cars.

Using a more or less sophisticated rendering technique depends on the application domain, where we must reach a compromise between speed and accuracy. An area where low rendering accuracy is sufficient is virtual environments where users interact with non-realistic virtual actors, as it has been proved that increasing rendering accuracy does not improve the quality of the perception 
of presence (Slater et al., 2001). Nevertheless, this kind of assertion may not be done for the rendering of urban environments. To sort out this, a new interesting research line would be to provide tools for tuning image realism in urban environments in order to highlight the real needs for synthetic images intended to improve driving simulators and security applications.

Despite managing urban scenes implies dealing with huge amounts of geometric data, the usability requirements of the application may permit to render with low image accuracy, so geometry can be simplified either in a preprocessing stage or on the fly during the rendering process. On another hand, objects closer or oriented toward the viewer require more details than others. So, different levels of accuracy will intervene in a single image. This introduces the well known Level of Detail (LoD) strategies which have been customized to urban scene geometry as well as to appearance models (Besuievsky and Patow, 2013; Clarberg et al., 2014). An alternative may be to think on the generation of a semantic LoD structure by means of associating different rendering parameters to the levels of another hierarchy. Then, the mixture of the different types of LoDs (geometry, rendering, and appearance) could be performed, offering a promising new focus to be explored. An example of appearance's LoD that could be further studied and customized to urban environments is a hierarchy of BRDFs (Tan et al., 2008).

Many urban modeling systems are procedural tools. This feature can also be exploited during the rendering phase. Two different approaches may be mentioned. A strategy described by Steinberger et al. (2014) is to generate just the geometry needed to produce each image, based on geometric criteria. Another way is proposed by Besuievsky et al. (2014), where the geometric elements are selected based on their sensitivity; i.e., depending on their effect on parameters like solar irradiation. Both approaches are opened to further research and analyzing the possible blending of them could lead on an original and powerful tool.

Graphics hardware (GPU) is used to perform many operations including rendering and model simplification (Steinberger et al., 2014). This resource is essential to increase the image realism, offering adequate modeling and rendering capabilities for many applications; but the degree of realism still remains far from the one obtained for indoor scenes and small outdoor ones in interactive applications. Even though some contributions (See Figure 1) presented global illumination approaches (Argudo et al., 2012), some problems are still open. We may group these problems in two categories: improving light transport mechanisms (LTM) and material effects (see Appearance Modeling). A research direction, we suggest to improve LTM is tailoring the concept of clustering in global illumination to urban environments, probably using a hierarchy of clusters.

\section{URBAN SIMULATIONS}

One of the areas where computer graphics may have its largest impact is on simulation quality, which would in turn result in a huge leap forward in other fields like comfort analysis, energy usage, and other positive aspects of urban life.

Urban Physics, in this context, refers to the study of a varied range of phenomena, like wind, sound, pollution, heat, illumination, and other phenomena that affect the living conditions in an urban landscape. Topics such as visibility and global illumination in urban areas were already discussed in Section "Rendering." However, all these effects correspond to the study of the short-wave range of the electromagnetic spectrum. On the other hand, the research community has just barely scratched the necessary methods for simulating longer wavelengths that represent heat transfer processes, mainly because of the inherent complexity of the simulation (Mirzaei and Haghighat, 2010). In spite of the large degree of attention that Finite-Element Methods (FEM) have received for solving differential equations, in computer graphics there is a large deal of experience in meshing and remeshing techniques, hierarchical methods, and clustering techniques.

Although some first steps have been given in urban climate studies, for instance by Aliaga et al. (2013), there is still much room for improvement. In general, Urban Physics deals with urban comfort, health and durability, referring respectively to pedestrian wind/thermal comfort, pollutant dispersion, and winddriven rain. For an in-depth survey of the discipline of Urban Physics, we refer the interested reader to the work by Moonen et al. (2012). In particular, the case of air pollution is of considerable importance (Vardoulakis et al., 2003), using numerical simulation methods to assess the pollution induced in air by factories or cars, and their impact on air quality in cities and rural landscapes. Although, to the best of our knowledge, there are no works in the field of computer graphics about these topics, we think meshing techniques and visualization tools can have a large impact on this area. A partial solution for this problem lies in the study of urban ecosystems, as already done by Beneš et al. (2011). In particular, procedural modeling techniques (Watson et al., 2008; Vanegas et al., 2010) would also have a large impact given its ability to quickly model a large number of buildings with the required level of detail, which is a fundamental aspect for the simulations involved in these studies.

\section{CONCLUSION}

In this paper, we have offered a perspective of some geometric and graphical tools that arise as outstanding opportunities to improve urban environments design. We have underlined aspects of geometric and appearance modeling, rendering, and simulation that influence the quality and usability of the designed Urban Environments. We have also described some of the limitations of existing tools and we have proposed new research directions to improve current state of the art for modeling and using Urban Environments, so potentially contributing to improve citizens life quality. These research lines are not limited to such environments, but will contribute to other applications of geometric modeling and image synthesis.

\section{AUTHOR CONTRIBUTIONS}

The contributions of all three authors have been equally distributed in the preparation of this manuscript.

\section{ACKNOWLEDGMENTS}

Funding: This work was partially funded by grant TIN201020590-C02-02 from Ministerio de Ciencia e Innovación, Spain, and by the European Commission under grant agreement FP7-IST-287639. 


\section{REFERENCES}

Advances in Architectural Geometry. (2014). Conference Series. Available at: http: //www.architecturalgeometry.org/aag14/

Aliaga, D. (2012). 3D design and modeling of smart cities from a computer graphics perspective. ISRN Comput. Graph. 2012, 728913. doi:10.5402/2012/728913

Aliaga, D. G., Vanegas, C., Lei, M., and Niyogi, D. (2013). Visualization-based decision tool for urban meteorological modeling. Environ. Plann. B Plann. Des. 40, 271-288. doi:10.1068/b38084

Argudo, O., Andújar, C., and Patow, G. (2012). "Interactive rendering of urban models with global illumination," in Computer Graphics International 2012, eds D. Thalmann, K. Zhou (Bournemouth: Springer Verlag).

Beneš, B., Massih, M. A., Jarvis, P., Aliaga, D. G., and Vanegas, C. A. (2011). "Urban ecosystem design," in Symposium on Interactive 3D Graphics and Games, I3D ‘11, eds C. Wyman, A. Varshney (New York, NY: ACM), 167-174.

Besuievsky, G., Barroso, S., Beckers, B., and Patow, G. (2014). "A configurable LoD for procedural urban models intended for daylight simulation," in Eurographics Workshop on Urban Data Modelling and Visualisation (Strasbourg), 19-24. doi:10.2312/udmv.20141073

Besuievsky, G., and Patow, G. (2013). Customizable lod for procedural architecture. Comput. Graph. Forum 32, 26-34. doi:10.1111/cgf.12141

Bosch, C., Laffont, P.-Y., Rushmeier, H., Dorsey, J., and Drettakis, G. (2011). Imageguided weathering: a new approach applied to flow phenomena. ACM Trans. Graph. 30, 20:1-20:13. doi:10.1145/1966394.1966399

Clarberg, P., Toth, R., Hasselgren, J., Nilsson, J., and Akenine-Möller, T. (2014). Amfs: adaptive multi-frequency shading for future graphics processors. ACM Trans. Graph. 33, 141. doi:10.1145/2601097.2601214

Dorsey, J., and Rushmeier, H. (2008). "Light and materials in virtual cities," in ACM SIGGRAPH 2008 Classes (Los Angeles, CA: ACM), 8:1-8:4. doi:10.1145/1401132. 1401143

Kuang, Z., Chan, B., Yu, Y., and Wang, W. (2013). A compact random-access representation for urban modeling and rendering. ACM Trans. Graph. 32, 172. doi:10.1145/2508363.2508424

Laffont, P.-Y., Bousseau, A., Paris, S., Durand, F., and Drettakis, G. (2012). Coherent intrinsic images from photo collections. ACM Trans. Graph. 31, 202:1-202:11. doi:10.1145/2366145.2366221

Lombardi, S., and Nishino, K. (2012). "Reflectance and natural illumination from a single image," in Computer Vision-ECCV 2012, eds A. Fitzgibbon, S. Lazebnik, P. Perona, Y. Sato and C. Schmid (Florence: Springer), 582-595. doi:10.1007/978-3-642-33783-3_42

Mirzaei, P. A., and Haghighat, F. (2010). Approaches to study urban heat island abilities and limitations. Build. Environ. 45, 2192-2201. doi:10.1016/j.buildenv. 2010.04.001

Moonen, P., Defraeye, T., Dorer, V., Blocken, B., and Carmeliet, J. (2012). Urban physics: effect of the micro-climate on comfort, health and energy demand. Front. Archit. Res. 1:197-228. doi:10.1016/j.foar.2012.05.002

Müller, P., Wonka, P., Haegler, S., Ulmer, A., and Van Gool, L. (2006). Procedural modeling of buildings. ACM Trans. Graph. 25, 614-623. doi:10.1145/1141911. 1141931

Musialski, P., Wonka, P., Aliaga, D., Wimmer, M., van Gool, L., and Purgathofer, W. (2013). A survey of urban reconstruction. Comput. Graph. Forum 32, 146-177. doi: $10.1111 /$ cgf.12077

Parish, Y. I. H., and Müller, P. (2001). "Procedural modeling of cities. In SIGGRAPH '01,' in Proceedings of the 28th Annual Conference on Computer Graphics and Interactive Techniques (New York, NY: ACM), 301-308. doi:10.1145/383259. 383292
Patow, G., and Besuievsky, G. (2013). "Challenges in procedural modeling of buildings," in Eurographics Workshop on Urban Data Modelling and Visualisation eds V. Tourre, G. Besuievsky (Girona: The Eurographics Association), 25-28.

SCENE. (2014). Novel Scene Representations for Richer Networked Media. Available at: http://3d-scene.eu

Slater, M., Steed, A., and Chrysanthou, Y. (2001). Computer Graphics and Virtual Environments: From Realism to Real-Time. Addison-Wesley.

Šlosár, P., Juránek, R., and Herout, A. (2014). "Cheap rendering vs. costly annotation: rendered omnidirectional dataset of vehicles," in Proceedings of the 30th Spring Conference on Computer Graphics, SCCG '14 (New York, NY: ACM), 71-78. doi:10.1145/2643188.2643191

Smelik, R. M., Tutenel, T., Bidarra, R., and Benes, B. (2014). A survey on procedural modelling for virtual worlds. Comput. Graph. Forum 33, 31-50. doi:10.1111/cgf.12276

Steinberger, M., Kenzel, M., Kainz, B., Wonka, P., and Schmalstieg, D. (2014). Onthe-fly generation and rendering of infinite cities on the gpu. Comput. Graph. Forum 33, 105-114. doi:10.1111/cgf.12315

Tan, P., Lin, S., Quan, L., Guo, B., and Shum, H.-Y. (2008). Filtering and rendering of resolution-dependent reflectance models. IEEE Trans. Vis. Comput. Graph 14, 412-425. doi:10.1109/TVCG.2007.70439

Vanegas, C. A., Aliaga, D. G., Wonka, P., Müller, P., Waddell, P., and Watson, B. (2010). Modelling the appearance and behaviour of urban spaces. Comput. Graph. Forum 29, 25-42. doi:10.1111/j.1467-8659.2009.01535.x

Vanegas, C. A., Garcia-Dorado, I., Aliaga, D. G., Benes, B., and Waddell, P. (2012). Inverse design of urban procedural models. ACM Trans. Graph. 31, 168. doi:10.1145/2366145.2366187

Vardoulakis, S., Fisher, B. E., Pericleous, K., and Gonzalez-Flesca, N. (2003). Modelling air quality in street canyons: a review. Atmos. Environ. 37, 155-182. doi:10.1016/S1352-2310(02)00857-9

Venkataraman, K., Lelescu, D., Duparré, J., McMahon, A., Molina, G., Chatterjee, P., et al. (2013). Picam: an ultra-thin high performance monolithic camera array. ACM Trans. Graph. 32, 166. doi:10.1145/2508363.2508390

Watson, B., Müller, P., Veryovka, O., Fuller, A., Wonka, P., and Sexton, C. (2008). Procedural urban modeling in practice. IEEE Comput. Graph. Appl. 28, 18-26. doi:10.1109/MCG.2008.58

Weber, B., Müller, P., Wonka, P., and Gross, M. H. (2009). Interactive geometric simulation of 4D cities. Comput. Graph. Forum 28, 481-492. doi:10.1111/j.14678659.2009.01387.x

Conflict of Interest Statement: The authors declare that the research was conducted in the absence of any commercial or financial relationships that could be construed as a potential conflict of interest.

Received: 30 July 2014; accepted: 05 December 2014; published online: 18 December 2014.

Citation: Pueyo X, Bosch C and Patow GA (2014) On improving urban environment representations. Front. Robot. AI 1:17. doi: 10.3389/frobt.2014.00017

This article was submitted to Virtual Environments, a section of the journal Frontiers in Robotics and AI.

Copyright (C) 2014 Pueyo, Bosch and Patow. This is an open-access article distributed under the terms of the Creative Commons Attribution License (CC BY). The use, distribution or reproduction in other forums is permitted, provided the original author(s) or licensor are credited and that the original publication in this journal is cited, in accordance with accepted academic practice. No use, distribution or reproduction is permitted which does not comply with these terms. 